\title{
The new landscape of medication adherence improvement: where population health science meets precision medicine
}

This article was published in the following Dove Press journal: Patient Preference and Adherence

\author{
Leah L Zullig',2
}

Dan V Blalock ${ }^{1,3}$

Samantha Dougherty ${ }^{4}$

Rochelle Henderson ${ }^{5}$

Carolyn $\mathrm{C} \mathrm{Ha}^{4}$

Megan M Oakes ${ }^{2}$

Hayden B Bosworth ${ }^{1-3,6}$

'Durham Center for Health Services Research in Primary Care, Durham Veterans Affairs Health Care System, Durham, NC, USA; ${ }^{2}$ Department of Population Health Sciences, Duke University, Durham, NC, USA;

${ }^{3}$ Department of Psychiatry and Behavioral Sciences, Duke University, Durham, NC, USA; ${ }^{4}$ Pharmaceutical Research and Manufacturers of America, Washington, DC, USA ${ }^{5}$ Express Scripts Holding Company, St Louis, MO, USA; ${ }^{6}$ School of Nursing, Duke University, Durham, NC, USA

Correspondence: Hayden B Bosworth Health Services Research and Development (I52), 4II W Chapel Hill Street, Suite 600, Durham, NC 2770I, USA

$\mathrm{Tel}+\mathrm{I} 9192866936$

Fax +19194165836

Email hayden.bosworth@duke.edu

\begin{abstract}
Despite the known health and economic benefits of medications, nonadherence remains a significant, yet entirely preventable public health burden. Over decades, there have been numerous research studies evaluating health interventions and policy efforts aimed at improving adherence, yet no universal or consistently high impact solutions have been identified. At present, new challenges and opportunities in policy and the movement toward value-based care should foster an environment that appreciates adherence as a mechanism to improve health outcomes and control costs (eg, fewer hospitalizations, reduced health care utilization). Our objective was to provide a commentary on recent changes in the landscape of research and health policy directed toward improving adherence and an actionable agenda to achieve system level savings and improved health by harnessing the benefits of medications. Specifically, we address the complementary perspectives of precision medicine and population health management; integrating data sources to develop innovative measurement of adherence and target adherence interventions; and behavioral economics to determine appropriate incentives.
\end{abstract}

Keywords: adherence, policy, precision medicine, population health

\section{The problem of medication nonadherence}

Medication nonadherence remains a substantial public health problem. Worldwide, between $25 \%$ and $50 \%$ of patients do not take their medications as recommended. ${ }^{1,2}$ In the USA, suboptimal adherence has been associated with 125,000 deaths, $10 \%$ of hospitalizations, and costs up to US\$289 billion annually. ${ }^{3-5}$ While medication adherence is a complex and multifaceted behavior, ${ }^{6}$ the negative public health effects of nonadherence are entirely preventable. There are a number of research studies that identify potential solutions to improve medication adherence incrementally. ${ }^{7-9}$ However, no universal or consistently high impact solutions have been identified. ${ }^{10,11}$ Additionally, in the overwhelmingly large academic literature on adherence interventions, relatively few studies simultaneously evaluate medication adherence and associated clinical outcomes (eg, adherence to anti-hypertensives and lower blood pressure). Moreover, when improvements are realized in both clinical and adherence outcomes, they tend to be of modest magnitude. ${ }^{12}$

While a broad scale medication adherence improvement solution remains elusive, many successful adherence interventions share common ingredients. According to our prior work, these factors include improving patients' understanding of their specific treatments, providing patients counseling and accountability, providing strategies and tools to help them self-monitor, and increase their access to medications. ${ }^{13}$ Despite 
having a relatively modest improvement in adherence for an individual patient, when interventions are scaled up to a population level there may be potential for significant improvement in public health (eg, a small increase in prescription refill rates could be associated with significant decreases in health care use). ${ }^{14}$ Yet even for promising strategies, only a small portion of research findings are translated to benefit patient care, and the time lag for implementation can be considerable. $^{15}$

\section{Research and health policy agenda to improve medication adherence}

In 2014, a research agenda to improve adherence was proposed by Seabury et al. ${ }^{16}$ In their commentary, Seabury et al defined six key areas to forward the medication adherence research agenda: 1) prediction of nonadherence, 2) behavioral factors affecting nonadherence, 3) measurement of the impact of nonadherence on health and cost outcomes, 4) effectiveness of existing interventions, 5) misaligned incentives between payers and providers, and 6) provider training and coordination of care. ${ }^{16}$ Since this publication, there has been increasing awareness in the topic of medication adherence, but little advancement in our understanding of how to realize the benefits of adherence. Additionally, there are new challenges and opportunities in the context of the evolving US health care system that impact adherence enhancing initiatives. As an example of the latter new challenges, Medicare Access and CHIP Reauthorization Act of 2015 (MACRA) and the movement toward value-based care should foster an environment that appreciates adherence as a mechanism to improve health outcomes.

Last year, the Pharmaceutical Research and Manufacturers of America (PhRMA) Foundation convened health policy and research experts representing patients, physicians, pharmacies, and the biopharmaceutical and insurance industries to gain consensus around priorities to address the adherence problem. ${ }^{17}$ Now, we take the next step to suggest several novel, targeted, and actionable research and policy agenda items to advance the field of medication adherence: 1) using a standardized taxonomy to describe adherence, adherencerelated study results, and quality reporting; 2) appreciating the complementary perspectives of precision medicine and population health management; 3 ) integrating data sources to develop innovative measurement of adherence and target recipients of adherence interventions (eg, targeting patients at high risk for hospitalization or rehospitalization); and 4) considering behavioral economics and determining the appropriate population and payer level incentives.

\section{Standardized taxonomy}

To support a stronger role of adherence in the broader goals of moving to a value-based health care system, there has been movement in the scientific literature to classify medication adherence into phases. The Ascertaining Barriers for Compliance (ABC) working group developed a taxonomy comprised of three quantifiable phases of adherence. ${ }^{6}$ The first is initiation. This phase is when a patient consumes a new medication as prescribed by their health care provider. The second phase of adherence is implementation. This phase involves when a patient's actual dose corresponds to the dosing that was prescribed from initiation until the last dose is taken. The third phase is discontinuation. This phase involves when a patient ceases taking their medication either independently or following their provider's instructions. ${ }^{6}$

Using a standardized taxonomy is helpful for advancing the field of medication adherence for a number of reasons. First, the taxonomy aids in more explicitly defining the nonadherence problem. Some data suggest that poor initiation, which is often not reported, may be a more concerning public health threat than implementation. Existing scientific literature generally reports information on adherence without the specificity of articulating what aspect of adherence is being studied. For example, many problems with patients not beginning a new therapy (ie, initiation) differ from problems with patients terminating therapy earlier than recommended (ie, discontinuation). Studying certain phases of adherence has historically been challenging because of data integration issues. For example, measuring initiation requires the linkage of prescription orders and prescription fill information, which are not commonly available in the same data set. This lack of consideration of medication adherence phases is problematic because there are nuances in the types of policies and interventional strategies that might be helpful to increase adherence at difference phases. For example, tools to aid shared decision making between prescribers and patients may be particularly relevant at the initiation phase. To support implementation, smartphone applications (apps) that provide medication dosing reminders may be useful. Without knowing which phase(s) are most problematic, it is challenging to know where to focus our efforts or apply the right solutions.

Second, few existing interventions have defined which phase(s) of adherence have been targeted. This makes it challenging to compare across studies and identify gaps in the scientific evidence. Most studies evaluate implementation (often using pharmacy-based measures such as proportion of days covered or medication possession ratio), 
perhaps because it is easier to measure. ${ }^{18}$ Measuring initiation may require linking nontraditional data sources such as e-prescribing information and pharmacy fill data. ${ }^{19,20}$ This linking of data is not commonly done outside of a select number of integrated health care systems. Similarly, identifying discontinuation in real time is challenging using electronic health record (EHR) and pharmacy data. Also challenging with EHR and pharmacy is determining where discontinuation is appropriate (ie, in accordance with a provider's instructions) or inappropriate (when a patient independently decides to stop taking a medication, perhaps due to side effects). Third, having a standard taxonomy facilitates the development of quality metrics which could be used to create incentive models related to improving medication adherence and monitoring progress over time.

There has been a move in the field to standardize definitions associated with adherence ${ }^{6}$ and, more recently, to create guidelines for reporting adherence-related interventions..$^{21} \mathrm{As}$ new studies begin to consistently use the same language and reporting standards, we will be better able to understand the landscape of adherence, quantify the problem with increased specificity, and identify adherence phase-relevant solutions to improve it.

\section{Complementary perspectives of precision medicine and population health}

In addition to using standard taxonomy, improving medication adherence requires considering the complementary perspectives of precision medicine and population health. In other words, tailored solutions addressing a patient's specific adherence barriers (precision medicine), scaled to the population level (population health), may be a successful strategy to facilitate improved medication adherence on a larger scale.

Precision medicine focuses on identifying patients with tailored and targeted intervention(s) delivered to meet their unique needs. The goal with precision medicine tends to have an individual impact. Currently, most literature reviews qualify "big impact" as an aggregate across many different patients, leading to only intensive and very complex interventions as demonstrating any real effectiveness at improving medication adherence. ${ }^{10,11}$ Furthermore, as indicated by a recent Cochrane review analysis, ${ }^{12}$ research often does not consider nonadherent individuals; in order to obtain clinical benefits from effective adherence interventions, researchers need to focus on the inclusion of nonadherent patients. Recent technological advancements in large national databases and data sharing, genomic sequencing, and predictive analytics have increased the feasibility and power of precision medicine, pushing precision medicine into the national spotlight as a presidentially driven research initiative. ${ }^{22}$ Similarly, increasing evidence demonstrates that incorporating individualized factors in medication use (eg, patient beliefs, goals, preferences, and specific patient barriers to medication taking) leads to both improved medication adherence and better clinical outcomes. ${ }^{23,24}$ Thus, combining the principles of patient-centered care with "big-data" and predictive analytic tools employed by precision medicine will allow researchers to identify patients at greatest risk for adherence problems, and allow clinicians to deliver adherence interventions to those who need it.

Population health, however, takes a broader approach to addressing medication adherence than precision medicine. Population health focuses on the health outcomes of a specified group of people, including the distribution of outcomes within that group. ${ }^{25}$ Therefore, population health might focus on delivering interventions to an entire patient group (ie, patients prescribed an anti-hypertensive medication) with the notion that small improvements across a broad population will have a large downstream impact on adherence and health outcomes among the patient population at large.

While precision medicine and population health take different approaches, there is a need for both. Aspects of precision medicine, such as tailoring therapies or intervention content to specific patients' needs, can be scaled up and applied in a population health model. For example, current population health models allow for distributions of outcomes to be conditioned among subpopulations, usually defined as geographic regions, but also occasionally defined as other groups, such as employees, insurance status, and disease groups, among others. Precision medicine techniques would allow for the development of other subgroups defined by a range of additional factors or unique combinations of factors (eg, unemployed individuals with co-occurring substance use problems; heart failure patients with stages 1-4 according to the New York Heart Association classification; forgetfulness), and the distribution of population-level factors such as copayments and outcomes could be examined along these subgroups as well. Enhancing the complementary perspectives of precision medicine and population health is critical; however, we assert the largest yield is with interventions and policies that are widely disseminated (Figure 1).

\section{Data integration}

In this climate of health care reorganization and uncertainly, there is a critical need for data integration. In the USA, 


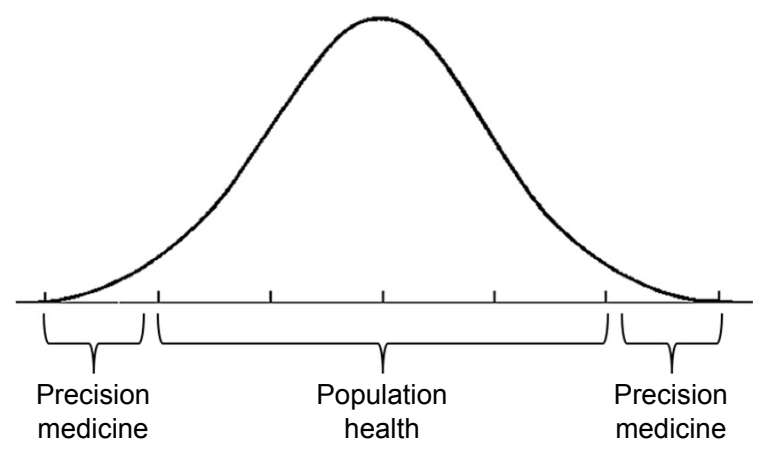

Figure I Complementary strengths of population health and precision medicine.

diagnosis and health care use information (eg, inpatient and outpatient electronic health record data) are generally created and managed by health care systems. Pharmacy fill and refill information (eg, retail and specialty pharmacy data) are typically created and managed by retail pharmacies and pharmacy benefit managers. Utilizing these large amounts of administrative data can yield interesting insights into broad medication nonadherence patterns, ${ }^{26}$ but triangulating information about adherence and linking it with clinical information may be challenging. Even in the case of Medicare and Medicaid, health care use and prescription medications are reimbursed through separate channels. For example, Medicare Parts A and B cover hospitalizations and medical care, whereas Part D covers medication benefits.

Data integration is important for several reasons. Clinically, it facilities better interprofessional communication among various health care providers, thus providing a more robust picture of the care being provided to a given patient. A robust picture of care can facilitate a precision medicine perspective by identifying patients with critical health risks who would benefit greatly from targeted adherence efforts. There are a few excellent examples of well integrated data from integrated health care systems such as Kaiser Permanente and Veterans Affairs. Because of data integration and accessibility, these health care systems are uniquely able to address research questions and inform the health care system in near real time. Specifically, the potential to reduce medication errors, reduce duplication of medications, and address seeking multiple providers with substance abuse disorders are some of the immediate benefits of additional adherence data integration. ${ }^{27}$

There is a need to follow this example with publically available data sources, such as Medicare Parts A, B, and D, in order to develop a robust understanding of adherence behaviors (eg, describe in context of the phases of adherence). Federal legislation has been introduced that would get at these linkages and data sharing. To enable health policy decisions to be informed by data, central to this effort is the need to use data interoperability standards and reduce delays in data reporting.

As technology advances, increasing incorporation of patient-generated self-monitoring data will likely increase. Furthermore, as many sources of innovative data become available, the need to integrate data will become even more important. For example, there is emerging digital chip technology that monitors when patients take their medication. The digital chip is embedded in a pill, which is activated upon contact with digestive enzymes in the stomach, and is read through a dermal patch. Information can be delivered to patients and providers in near real time. This is currently being used for patients diagnosed with schizophrenia. Once provided with objective information about a patient's adherence, their provider can determine if oral medication or a long-acting injectable is preferable. ${ }^{28}$

\section{Behavioral science and incentivizing medication adherence}

Reinforcement interventions, programs that are based on behavioral economic principles, are becoming more widely used in clinical practice. Behavioral economic principles have guided financial and social incentive structure programs designed to address a patient's propensity for nonadherence. A recent review found that both randomized studies and nonrandomized studies evaluated the efficacy of financial reinforcement to improve medication adherence. ${ }^{29}$ Financial reinforcement interventions apply behavioral economic principles by which a patient received monetary compensation for meeting health goals. Across the reviewed studies, financial incentives were applied for adherence to medications in several disease states. Reinforcement interventions meaningfully improved adherence and did so with a medium to large overall effect size. Compared to randomized studies, on average, the effect size was larger in nonrandomized studies. The studies that demonstrated the largest effect sizes were longer, provided hefty financial incentives ( $\geq$ US $\$ 50$ weekly), and interacted with patients often (at least weekly). ${ }^{29}$

In efforts to improve system financial incentives by linking payment to value rather than volume, some pharmaceutical manufacturers and private payers have shown an interest in outcomes-based contracts. These contracting arrangements tie the price paid for drugs to their effectiveness in treating targeted patients, allowing manufacturers to share risk for the medicine's performance in the payer's population. By reducing the payer's risk associated with the medicine not 
performing as expected, outcomes-based contracting could help support appropriate access to medications, potentially impact out-of-pocket prices for patients, and reduce payers' concerns about wasting resources on medications that do not perform as expected outside of clinical trials or for select subpopulations.

Another behavioral economic program is value-based insurance design (V-BID). V-BID relies on insurance costsharing. Patients pay a reduced price for medications that have a higher clinical value. A review of 10 studies that used V-BID medication adherence improved adherence between 2 and 5 percentage points across the included studies. While medication adherence improved, it was uncertain whether the associated clinical outcomes, health care use, and health care spending also improved. ${ }^{30}$

Few studies have looked at long-term use of behavioral economic principles. A key aspect of behavioral economics is that people place a disproportionate emphasis on immediate outcomes and do not fully consider possible, downstream, or delayed events. ${ }^{31}$ In the context of medication adherence, patients may consider short-term inconveniences associated with taking a pill or managing unpleasant side effects and may disregard or not value the longer-term outcomes of potential for disability or even death. In other words, eliminating copayments will likely not completely resolve nonadherence in people who miss medications, for example, due to beliefs about side effects. Helping patients focus on direct and immediate positive outcomes of taking their medication through the use of financial incentives has the potential to improve both individual and population health.

\section{Conclusion}

Medication adherence remains at the forefront of public health burdens facing the USA. Improving adherence requires a multifaceted approach, engaging principles of both precision medicine and population health. We assert that innovative approaches are needed to advance the field of medication adherence including embracing a standardized taxonomy to describe adherence and report adherence-related study results, integrating data sources to develop innovative measurement of adherence, and considering innovative mechanisms (ie, behavioral economics principles) to incentivize both patients and payers to engage in improving medication adherence.

\section{Acknowledgments}

This work was supported by the Center of Innovation for Health Services Research in Primary Care (CIN 13-410) at the Durham Veterans Affairs (VA) Medical Center and the PhRMA Foundation. We thank Eileen Cannon, President of the PhRMA Foundation, for her support.

\section{Disclosure}

Dr Zullig is supported by VA Health Services Research and Development (HSR\&D) Career Development Awards (CDA 13-025). Dr Bosworth is supported by a Career Scientist award (RCS 08-027). Dr Blalock was supported by Grant No TPH 21-000 from the Department of Veterans Affairs Office of Academic Affiliations. The authors report no other conflicts of interest in this work.

\section{References}

1. Sabaté E. Adherence to Long-Term Therapies: Evidence for Action. Geneva: World Health Organization; 2003.

2. van Dulmen S, Sluijs E, van Dijk L, de Ridder D, Heerdink R, Bensing J Patient adherence to medical treatment: a review of reviews. $B M C$ Health Serv Res. 2007; 7:55.

3. Osterberg L, Blaschke T. Adherence to medication. $N$ Engl J Med. 2005;353(5):487-497

4. Peterson AM, Takiya L, Finley R. Meta-analysis of trials of interventions to improve medication adherence. AJHP. 2003;60(7):657-665.

5. Viswanathan M, Golin CE, Jones CD, et al. Interventions to improve adherence to self-administered medications for chronic diseases in the United States: a systematic review. Ann Intern Med. 2012;157(11) 785-795.

6. Vrijens B, De Geest S, Hughes DA, et al. A new taxonomy for describing and defining adherence to medications. Br J Clin Pharmacol. 2012; 73(5):691-705.

7. Adler AJ, Martin N, Mariani J, et al. Mobile phone text messaging to improve medication adherence in secondary prevention of cardiovascular disease. Cochrane Database Syst Rev. 2017;4:Cd011851.

8. Normansell R, Kew KM, Stovold E. Interventions to improve adherence to inhaled steroids for asthma. Cochrane Database Syst Rev. 2017;4:Cd012226.

9. van Driel ML, Morledge MD, Ulep R, Shaffer JP, Davies P, Deichmann R. Interventions to improve adherence to lipid-lowering medication. Cochrane Database Syst Rev. 2016;12:Cd004371.

10. Haynes RB, Ackloo E, Sahota N, McDonald HP, Yao X. Interventions for enhancing medication adherence. Cochrane Database Syst Rev. 2008;(2):CD000011.

11. Nieuwlaat R, Wilczynski N, Navarro T, et al. Interventions for enhancing medication adherence. Cochrane Database Syst Rev. 2014;11: CD000011.

12. Allemann SS, Nieuwlaat R, Navarro T, Haynes B, Hersberger KE, Arnet I. Congruence between patient characteristics and interventions may partly explain medication adherence intervention effectiveness: an analysis of 190 randomized controlled trials from a Cochrane systematic review. J Clin Epidemiol. 2017;91:70-79.

13. Zullig LL, Peterson ED, Bosworth HB. Ingredients of successful interventions to improve medication adherence. JAMA. 2013;310(24): 2611-2612.

14. Krumme AA, Glynn RJ, Schneeweiss S, et al. Medication synchronization programs improve adherence to cardiovascular medications and health care use. Health Aff. 2018;37(1):125-133.

15. Lenfant C. Shattuck lecture - clinical research to clinical practice-lost in translation? N Engl J Med. 2003;349(9):868-874.

16. Seabury SA, Gupta CN, Philipson TJ, Henkhaus LE. Understanding and overcoming barriers to medication adherence: a review of research priorities. J Manag Care Spec Pharm. 2014;20(8):775-783. 
17. Bosworth H, Salzburg S. Why Medication Adherence Needs To Be A National Priority. 2017. Available from: https://www.forbes.com/ sites/sciencebiz/2017/06/12/why-medication-adherence-needs-to-bea-national-priority/2/-2b7c25ee5279. Accessed February 8, 2018.

18. Zullig LL, Ramos K, Bosworth HB. Improving medication adherence in coronary heart disease. Curr Cardiol Rep. 2017;19(11):113.

19. Fallis BA, Dhalla IA, Klemensberg J, Bell CM. Primary medication non-adherence after discharge from a general internal medicine service. PLoS One. 2013;8(5):e61735.

20. Harnett J, Wiederkehr D, Gerber R, Gruben D, Bourret J, Koenig A. Primary nonadherence, associated clinical outcomes, and health care resource use among patients with rheumatoid arthritis prescribed treatment with injectable biologic disease-modifying antirheumatic drugs. J Manag Care Spec Pharm. 2016;22(3):209-218.

21. Helmy R, Zullig LL, Dunbar-Jacob J, et al. ESPACOMP Medication Adherence Reporting Guidelines (EMERGE): a reactive-Delphi study protocol. BMJ Open. 2017;7(2):e013496.

22. Collins FS, Varmus H. A new initiative on precision medicine. $N$ Engl J Med. 2015;372(9):793-795.

23. Kangovi S, Mitra N, Grande D, et al. Patient-centered community health worker intervention to improve posthospital outcomes: a randomized clinical trial. JAMA Intern Med. 2014;174(4):535-543.

24. van Boven JF, Stuurman-Bieze AG, Hiddink EG, Postma MJ, Vegter S. Medication monitoring and optimization: a targeted pharmacist program for effective and cost-effective improvement of chronic therapy adherence. J Manag Care Spec Pharm. 2014;20(8):786-792.
25. Kindig D, Stoddart G. What is population health? Am J Public Health. 2003;93(3):380-383.

26. Karter AJ, Parker MM, Moffet HH, Ahmed AT, Schmittdiel JA, Selby JV. New prescription medication gaps: a comprehensive measure of adherence to new prescriptions. Health Serv Res. 2009;44(5p1): 1640-1661.

27. Hughes R. Patient Safety and Quality: An Evidence-Based Handbook for Nurses. Vol 3. Rockville, MD: Agency for Healthcare Research and Quality; 2008.

28. Belluck P. First Digital Pill Approved to Worries About Biomedical "Big Brother". 2017. Available from: https://www.nytimes.com/ 2017/11/13/health/digital-pill-fda.html. Accessed February 9, 2018.

29. Petry NM, Rash CJ, Byrne S, Ashraf S, White WB. Financial reinforcers for improving medication adherence: findings from a meta-analysis. Am J Med. 2012;125(9):888-896.

30. Tang KL, Barnieh L, Mann B, et al. A systematic review of valuebased insurance design in chronic diseases. Am J Manag Care. 2014; 20(6):e229-e241.

31. Mischel W, Shoda Y, Rodriguez MI. Delay of gratification in children. Science. 1989;244(4907):933-938.
Patient Preference and Adherence

\section{Publish your work in this journal}

Patient Preference and Adherence is an international, peer-reviewed, open access journal that focuses on the growing importance of patient preference and adherence throughout the therapeutic continuum. Patient satisfaction, acceptability, quality of life, compliance, persistence and their role in developing new therapeutic modalities and compounds to optimize

\section{Dovepress}

clinical outcomes for existing disease states are major areas of interest for the journal. This journal has been accepted for indexing on PubMed Central. The manuscript management system is completely online and includes a very quick and fair peer-review system, which is all easy to use. Visit http://www. dovepress.com/testimonials.php to read real quotes from published authors. 